\title{
GENUS Didymocarpus Wall. AND A NEW RECORD OF SPECIES Didymocarpus purpureobracteatus Smith FOR THE FLORA OF VIETNAM FROM XUAN LIEN NATURAL RESERVE
}

\author{
Vu Xuan Phuong ${ }^{1}$, Dang Quoc Vu ${ }^{2}$, Do Thi Xuyen ${ }^{3 *}$ \\ ${ }^{1}$ Institute of Ecology and Biological Resources, VAST \\ ${ }^{2}$ Forest Protection Department, Ministry of Agriculture and Rural Development \\ ${ }^{3}$ VNU University of Science, *xuyendoiebr@gmail.com
}

\begin{abstract}
Species Didymocarpus purpureobracteatus Smith is a new record for the flora of Vietnam. It was regarded as one endemic species to China, but up to now we have found it in Xuan Lien natural reserve (Thanh Hoa province). Voucher specimens were deposited in the Herbarium of the Institute of Ecology and Biological Resources, Hanoi, Vietnam (HN). This species is closed to $D$. poilanei but differs by bract elliptic or ovate, ca. 3-8 $\mathrm{mm}$ long; sepal tube ca. $8-9 \mathrm{~mm}$ long, sepal lobed ca. 2-3 mm long. So far, in Vietnam there were 5 species of the genus Didymocarpus Wall. recorded D. bonii, D. pulcher, D. kerrii, D. poilanei and D. purpureobracteatus.

Keywords: Gesneriaceae, Didymocarpus, new record, Xuan Lien natural reserve, Vietnam.
\end{abstract}

\section{INTRODUCTION}

According to Wang et al. 1998 [10], the genus Didymocarpus Wall. (Gesneriaceae) comprises about 180 species, mainly distributed South-East Asia, there were 4 Didymocarpus species recorded in Vietnam [8]. During the study of specimens and documents of Gesneriaceae of Vietnam, we found Didymocarpus: Didymocarpus purpureobracteatus Smith collected in Xuan Lien reserver, Thanh Hoa province, Vietnam. Therefore, this is a new record for the flora of Vietnam and to date the genus Didymocarpus has 5 species. In this paper, we provide the key to these species of the genus Didymocarpus, some new information of these species and characteristic morphology for finding $D$. purpureobracteatus in Vietnam.

\section{MATERIALS AND METHODS}

All specimens of Didymocarpus were collected in Vietnam, including specimens in herbarium of Institute of Ecology and Biological Resources (IEBR), Institute of Biological Tropical (VNM), National Institute of Medicinal Material (HNPM), Vietnam National University, Hanoi (HNU), Kunming Institute of Botany, China (KUN), Kwangxi Institute of Botany, China (KIB), South Botany China Institute, China (SBCI), National Natural Museum of History Paris, France $(\mathrm{P})$ and fresh plants in the field.

The comparative morphology methods is used for the study. This is traditional method, which is usually used in classification of plant. All drawings made by Mrs Ly Kim Chi (10-19) and Mr Nguyen Quang Hung (1-9) (IEBR).

\section{RESULTS AND DISCUSSION}

\section{Taxonomy key to the species of genus Didymocarpus Wall. in Vietnam}

1A. Sepal actinomorphic, 5-sect from base; filament with glandular hair.. 1. D. bonii

1B. Sepal zygomorphic, 5-lobed into 2 lips, 1 lip 3-lobed, 1 lip 2-lobed, filament smooth.

2A. Ovary smooth.

3A. Corolla outer hair; leaf blade ovate or elliptic, ca. 4-10 $\times 2-6 \mathrm{~cm}$ ..2. D. pulcher

3B. Corolla outer smooth; leaf blade near round or broadlly elliptic, ca. 1-4,5 $\times 1-4 \mathrm{~cm}$....3. D. kerrii 
2B. Ovary hair.

4A. Base of leaf often axisymmetric; bract lanceolata, ca. 2-3 mm long; sepal tube ca. $3 \mathrm{~mm}$ long, sepal lobed ca. 1-1,5 mm long. 4. D. poilanei

4B. Base of leaf often oblique; bract elliptic or ovate, ca. 3-8 mm long; sepal tube ca. 8-9 mm long, sepal lobed ca. 2-3 mm long......

5. D. purpureobracteatus

Some new information of species Didymocarpus and chracteristic morphology of $D$. purpureobracteatus - a new record for the flora of Vietnam.

1. Didymocarpus bonii Pellegr. - Song quả bon (Fig. 1-4)

Pellegr. 1926. Bull. Soc. Bot. France, 73: 416; id. 1930. Fl. Gen. Indoch. 4: 520; Phamh. 1993. Illustr. Fl. Vietn. 3: 11; V. X. Phuong, 2005. Checkl. Pl. Sp. Vietn. 3: 242.

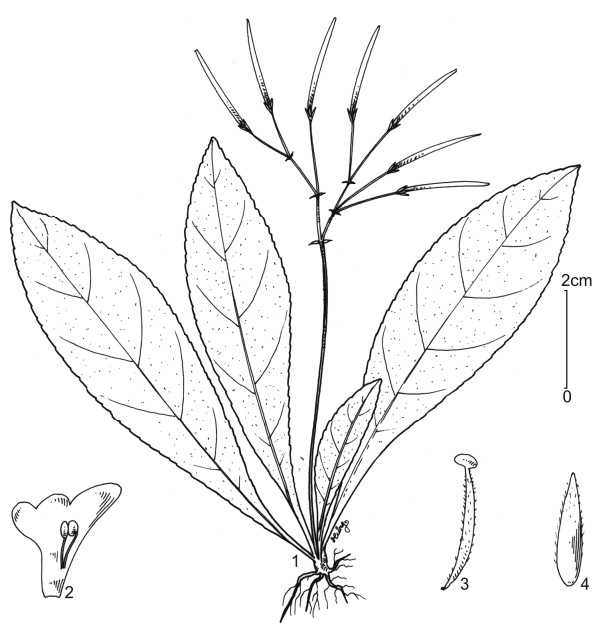

Fig 1-4. Didymocarpus bonii Pellegr.

1. plant; 2. corolla opened; 3 . stamen; 4 . bract

(Specimen Bon 5730, P)

Diagnostic morphology: Sepal actinomorphic, 5-sect from base; filament with glandular hair.

Loc.class.: Indochine, Tonkin: Matson. Typus: Bon $5730(\mathrm{P})$.

Ecology: Flower and fruit in June-October. In limestone mountains, cliff, small cave in the rocky mountain.

Distribution: Only in the North of Vietnam: Ninh Binh (Cho Ghenh), Thanh Hoa (Mat Son).

Specimens examined: Ninh Binh, Petelot 959 (P). Thanh Hoa, Bon 5730 (P).
2. Didymocarpus pulcher C. B. Clarke - Song quả pulcher (Fig. 5-7)

C. B. Clarke, 1883. Monogr. Phan. 5: 79; id. 1884. Fl. Brit. Ind. 4: 348; Pellegr. 1930. Fl. Gen. Indoch. 4: 523; W. T. Wang, 1990. Fl. Reip. Pop. Sin. 69: 434; Phamh, 1993. Illsstr. Fl. Vietn. 3: 12; W. T. Wang, 1998. Fl. China, 18: 354; V. X. Phuong, 2005. Checkl. Pl. Sp. Vietn. 3: 242.

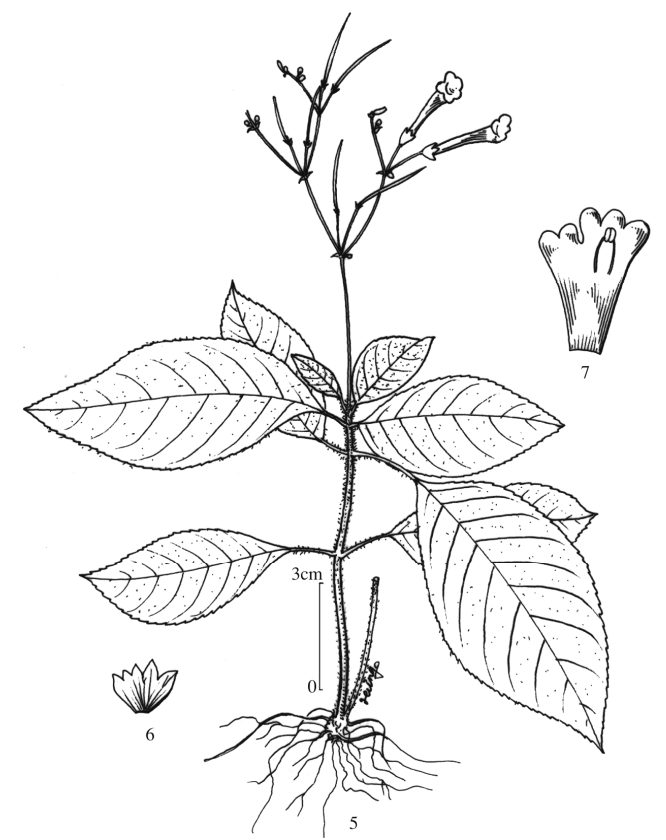

Fig 5-7. Didymocarpus pulcher C. B. Clarke 5. plant; 6. sepal opened; 7. corolla opened

(Specimen LX-VN 111, HN)

Diagnostic morphology: Leaf blade ovate or elliptic, ca. 4-10 $\times 2-6 \mathrm{~cm}$; sepal zygomorphic, 5-lobed into 2 lips, 3/2; corolla outer hair; filament smooth; ovary smooth.

Loc.class.: Himalaya centralis, Sikkim. Typus: Griffith3841 (K).

Ecology: Flower and fruit in June-August. In every green forest, soil mountains and 
limestone mountains, at 600-1,000 m alt..

Distribution: Lao Cai (Sa Pa), Quang Ninh (Ha Long bay), Vinh Phuc (Tam Dao national park), Ha Noi (Ba Vi national park), Thanh Hoa (Ba Thuoc, $\mathrm{Pu}$ Luong natural reserve), Thua Thien-Hue (Bach Ma national park), Quang Nam (Song Thanh natural reserve). And India, Nepal, China.

Specimens examined: Lao Cai, Petelot 8699 (HM); TV 359 (HN); HNK 469 (HN). Vinh Phuc, LX-VN 74 (HN); $111(\mathrm{HN}) ; 327$ (HN); N. T. Hiep 14980 (HN); H. V. Ve 15022 (HN); T. D. Nghia T013 \& 263 (HNU). - Hanoi, Balansa 4295 (P). - Thanh Hoa, HAL 4197 (HN). - Thua Thien-Hue, TVC 527 (HN). Quang Nam, TVC 487 (HN).

\section{Didymocarpus kerrii Craib - Song quả kerri (Fig. 8-9)}

Craib, 1911. Kew Bull. 1911: 431; id. 1912. Contrib. Fl. Siam. 149; Pellegr. 1930. Fl. Gen. Indoch. 4: 525; V. X. Phuong \& D. T. Xuyen, 2012. Journ. Bot. 34(1): 72-74.

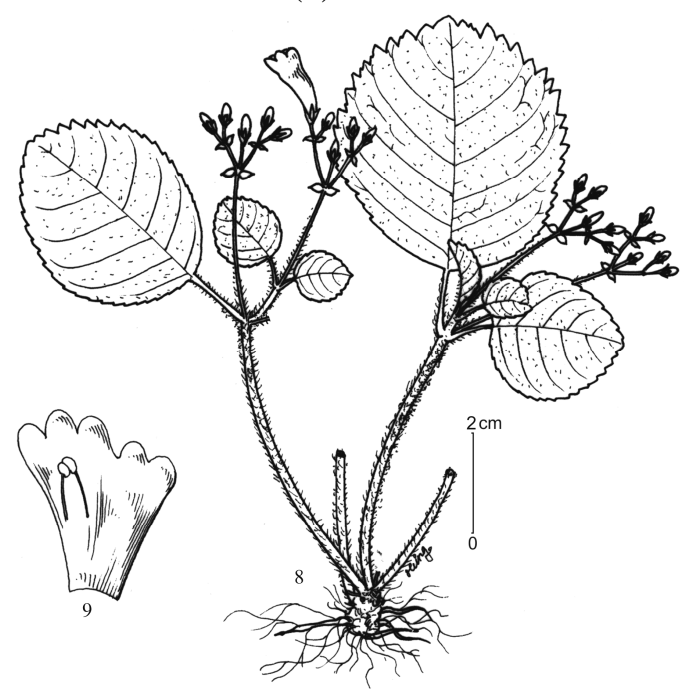

Fig 8-9. Didymocarpus kerrii Craib

8. plant; 9. corolla opened

(Specimen N. K. Dao 14156, HN)

Diagnostic morphology: Leaf blade near round or broadlly elliptic, ca. 1-4.5 × 1-4 cm; Sepal zygomorphic, 5-lobed into 2 lips, 3/2; corolla outer smooth; filament smooth; ovary smooth.
Loc.class.: Siam, Chiengmai (Doi Sootep). Typus: Kerri 786 (K).

Ecology: Flower and fruit in March-June. In every green forest, soil mountains and limestone mountains, at under $1600 \mathrm{~m}$ about the sea.

Distribution: Hanoi (Ba Vi), Thailand.

Specimen examined: Hanoi, Werff \& N. K. Dao 14156 (HN).

\section{Didymocarpus poilanei Pellegr. - Song quả poilane (Fig. 10-14)}

Pellegr. 1930. Fl. Gen. Indoch. 4:523; Phamh. 1993. Illust. Fl.Vietn.3:11; V. X. Phuong, 2005. Checkl. Pl. Sp. Vietn. 3: 242.

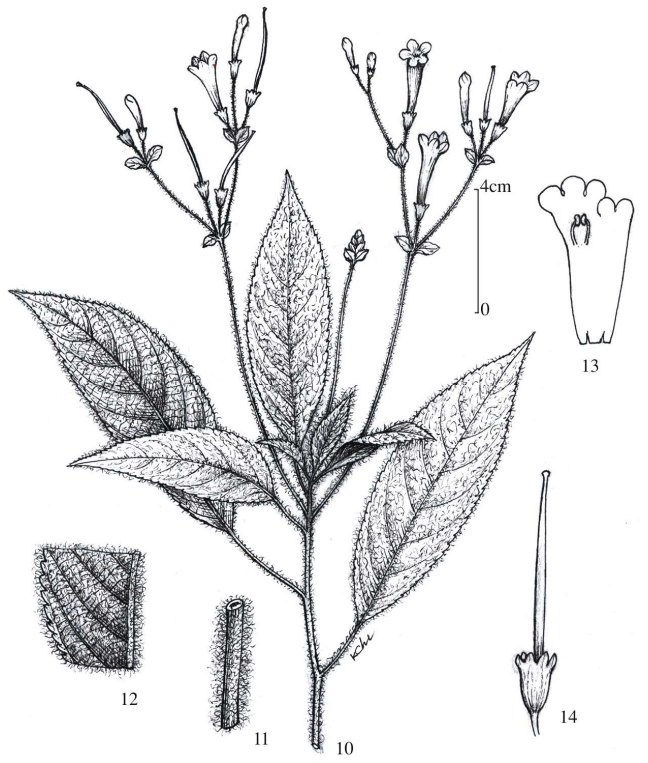

Fig 10-14. Didymocarpus poilanei Pellegr. 10. plant; 11. pieces of stem; 12. pieces of leaf; 13. corolla opened; 14. sepal and style

$$
\text { (Specimen VH 4297, HN) }
$$

Diagnostic morphology: Base of leaf often axisymmetric; bract lanceolata, ca. 2-3 $\mathrm{mm}$ long; sepal zygomorphic, 5-lobed into 2 lips, $3 / 2$, sepal tube ca. $3 \mathrm{~mm}$ long, sepal lobed ca. 11,5 mm long; filament smooth; ovary hair.

Loc.class.: Annam, Nha Trang. Typus: Poilane 3417 (P).

Ecology: Flower and fruit in MaySeptember. In every green forest, soil mountains and limestone mountains, at 800$1200 \mathrm{~m}$ about the sea. 
Distribution: Only in central of Vietnam: Thua Thien-Hue (A Luoi), Khanh Hoa (Nha Trang).

Specimens examined: Thua Thien-Hue, T. M. Quang 47 (HN). - Khanh Hoa, Poilane 3417 (P); VH 4297 (HN).

\section{Didymocarpus purpureobracteatus Smith - Song quả lá bắc tím}

Smith, 1912. Notes Roy. Bot. Gard. Edinb.5: 153, Pl.107; W. T. Wang, 1990. Fl. Reip. Pop. Sin. 69: 441; id. 1998. Fl. China, 18: 356.

- Didymocarpus veitchianus Smith, 1912. 1. c. 5: 154.; Didymocarpus purpureobracteatus var. veitchianus (Smith) H. W. Li, 1983. Bull. Bot. Res. 3(2): 32 .

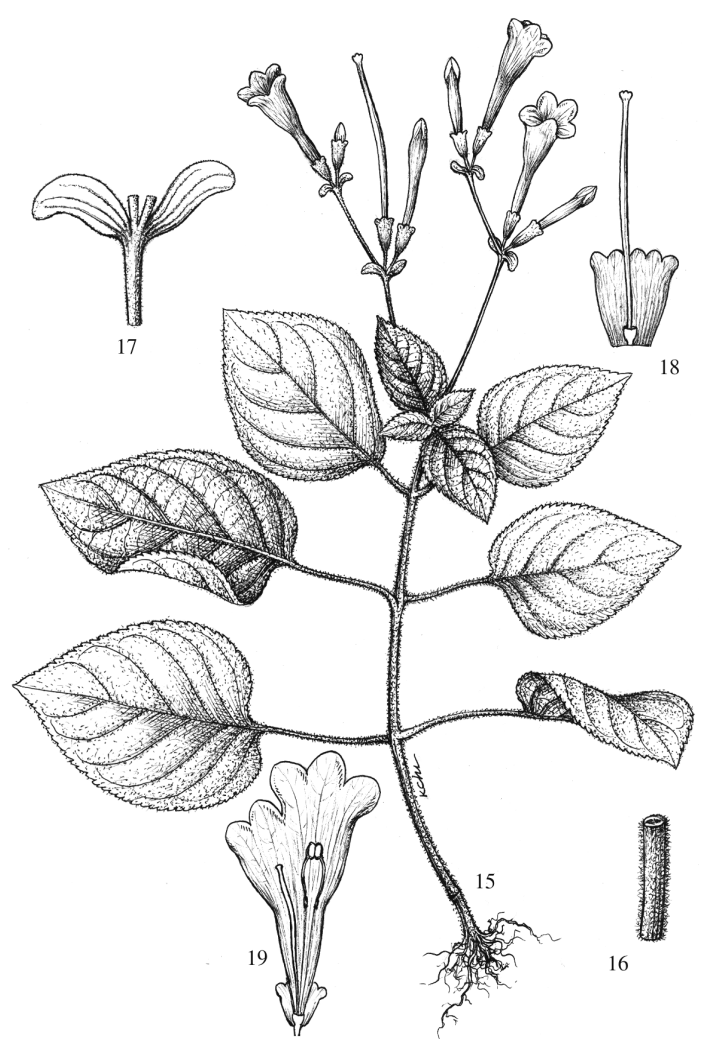

Fig 15-19. Didymocarpus purpureobracteatus Smith.

15. plant; 16. pieces of stem; 17 . bract; 18 . sepal opened and style; 19 . flower opened

(Specimen XL 553, HN)
Morphology: Stems 10-60 cm, young part sparsely puberulent. Leaves opposite; leaf blade ovate to obovate, $3-17(-25) \times 1.5-10(-15) \mathrm{cm}$, apex acute to acuminate, base often oblique, round to cordate; margin minute serrate; 2 surface sparsely appressed puberulent to glabrous, sparsely glandular; lateral veins 5-9 on each side of midrib; petiole 1-10 cm. Cymes often 5-10 flowered in axil; peduncle $4-10 \mathrm{~cm}$, puberulent to glabrous; bracts purple, ovate to elliptic-ovate, 3-8 $\mathrm{mm}$ long, glabrous, margin entire. Pedicel 1-5 mm. Calyx slightly zygomorphic, 1-1.2 cm long; limb indistinctly 2-lipped; tube 8-9 mm; adaxial lip 3-lobed, 2-3 $\times 3 \mathrm{~mm}$, lobes semiorbicular; abaxial lip 2lobed, 2-3 × 4-5 mm, lobes semiorbicular, glabrous. Corolla purple to pinkish purple, ca. 3-4 cm, glabrous; tube funnelform, ca. 2.5-3.0 long, 2 lips; adaxial lip 3-4 mm long; abaxial lip 8-10 mm long. Stamen 2, Filaments ca. $1 \mathrm{~cm}$, glabrous; anthers ca. $3 \mathrm{~mm}$, puberulent; staminodes 2, 1-2 mm. Pistil 2.1-2.5 cm, sparsely puberulent; ovary ca. $2-2.3 \mathrm{~cm}$. Capsule 3-5 cm. Seed unappendage.

Loc. class.: China, Yunnan.

Typus: A. Henry 9189 (K).

Ecology: Flower and fruit in JulySeptember. In evergreen forest, soil mountains and limestone mountains, 800- $1200 \mathrm{~m}$ alt..

Distribution: Thanh Hoa (Xuan Lien natural reserve). China (SE Yunnan).

Specimen examined: THANH HOÁ, XL 553: N $19^{0}$ 53'33.1',; E $105^{\circ} 10^{\prime} 54.1^{\prime}$ ', at 1427 $\mathrm{m}$ near the sea $(\mathrm{HN})$.

Notes: This species is closed to D. poilanei but differs by bract elliptic or ovate, ca. 3-8 $\mathrm{mm}$ long; sepal tube ca. 8-9 mm long, sepal lobed ca. 2-3 mm long.

Acknowledgements: This study was supported by funds from the program VAST 04.08/12-13 of Vietnam Academy Science and Technology. The authors would like to thank this program and herbaria HN, HNPM, HNU, IBK, IBSC, KUN, P, VNM. We also thank painter Le Kim Chi, Msc. Do Van Hai, Dr. Nguyen The Cuong, PhD. student Vu Tien Chinh and Xuan Lien 
natural reserve, and others for providing some materials and creating opportunity for our study.

\section{REFERENCES}

1. Backer C. A., Bakhuizen C. R., 1965. Flora of Java, 2: 518-534. Netherlands.

2. Burtt B. L., 1977. Notes Roy. Bot. Gard. Edinburgh, 36(1): 151-155.

3. Burtt B. L., 2001. Thai Forest Bull. Bot. 29: 81-109.

4. Davidson R., Burtt B. L., 1954. Notes Roy. Bot. Gard. Edinburgh, 21(4): 193-208.

5. Pham Hoang Ho, 2000. An Illustrated flora of Vietnam, 3: 12-29. Tre publishing house,
Ho Chi Minh city.

6. Pan K. Y. in Wang W. T., Pan K. Y., Li Z. Y., 1990. Flora Reipublicae Popularis sinicae, 69: 125- 581. Science Press, Beijng.

7. Pellegrin F. in H. Lecomte, 1930. Flore générale de L'Indo-chine, 4: 487-565. Paris.

8. Vu Xuan Phuong, Do Thi Xuyen, 2012. A new occurence species Didymocarpus kerrii Craib (Gesneriaceae Dumort) of the flora of Vietnam. Journal of Botany, 34(1): 72-74.

9. Wang W. T., 1984. Notulae de Gesneriaceis Sinensibus. Bull. Bot. Res., 4(1): 9-35.

10. Wang W. T. et al., 1998: Gesneriaceae Dumort. Flora of China, 18: 367-368. USA.

\title{
CHI SONG QUẢ (Didymocarpus) VÀ BỔ SUNG LOÀI Didymocarpus purpureobracteatus Smith CHO HÊ THƯC VẠT VIẸT NAM TỬ KHU BẢO TỒN THIÊN NHIÊN XUÂN LIỀN, TỈNH THANH HÓA
}

\author{
Vũ Xuân Phương ${ }^{1}$, Đặng Quốc Vũ̃ , Đỗ Thị Xuyến ${ }^{3}$ \\ ${ }^{1}$ Viện Sinh thái và Tài nguyên sinh vật, Viện Hàn lâm KH \& CN Việt Nam \\ ${ }^{2}$ Cục Kiểm lâm, Bộ Nông nghiệp và Phát triển nông thôn \\ ${ }^{3}$ Trường Đại học Khoa học tự nhiên, ĐHQG Hà Nội
}

\section{TÓM TẮT}

Ghi nhận mới loài Didymocarpus purpureobracteatus Smith - Song quả lá bắc tím cho hệ thực vật Việt Nam. Đây là loài cây thảo, trước kia được coi là đặc hữu của Trung Quốc, đến nay chúng tôi đã tìm thấy có ở Khu BTTN Xuân Liên, tỉnh Thanh Hoá. Mẫu vật hiện đang được lưu giữ tại Phòng tiêu bản thực vật của Viện Sinh thái và Tài nguyên sinh vật $(\mathrm{HN})$. Như vậy, cho đến nay, chi Didymocarpus Wall. ở Việt Nam hiện được ghi nhận có 5 loài là $D$. bonii, $D$. pulcher, $D$. kerrii, $D$. poilanei và $D$. purpureobracteatus. Bài báo đã đưa ra khóa định loại cho 5 loài thuộc chi này ở Việt Nam.

Loài Song quả lá bắc tím - Didymocarpus purpureobracteatus Smith có các đặc điểm dễ nhận dạng như: thân dạng cỏ, cao 10-60 cm, có lông rải rác ở phần non. Lá hình trứng hay trứng ngược, gốc thường bất xứng, mép có răng, cuống lá dài $1-10 \mathrm{~cm}$. Cụm hoa hình xim mọc ở nách lá, thường mang 5-10 hoa, cuống cụm hoa dài 4-10 cm; lá bắc màu tím, hình trứng tới bầu dục-trứng, dài 3-8 mm, nhẵn, mép nguyên; cuống hoa dài 1-5 $\mathrm{mm}$; đài đối xứng hai bên, dài 1-1,2 $\mathrm{cm}$; chia 2 môi; ống đài dài $8-9 \mathrm{~mm}$; thùy đài thường có chóp gần tròn, dài 2-3 mm; tràng màu đỏ tía tới hồng nhạt, dài $3-4 \mathrm{~cm}$. Nhị hữu thụ 2 , chỉ nhị dài đến $10 \mathrm{~mm}$; nhị bất thụ 2 ngắn dưới $1 \mathrm{~mm}$. Quả nang dài $3-5 \mathrm{~cm}$. hạt không có phần phụ. Loài này rất gần với loài $D$. poilanei nhưng khác bởi đặc điểm rất dễ nhận là lá bắc to, hình bầu dục hay hình trứng, dài 3-8 mm; ống đài dài $8-9$ mm, thùy đài dài 2-3 mm.

Tù khóa: Gesneriaceae, Didymocarpus, D. purpureobracteatus, song quả, Xuân Liên.

Ngày nhận bài: 15-5-2013 